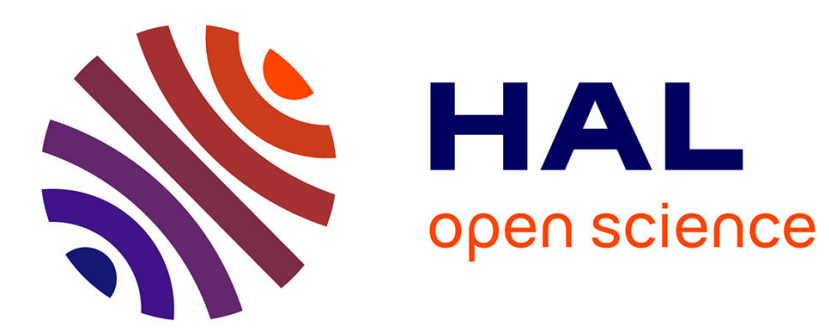

\title{
Basic mechanisms of, and treatment planning/targets for, obsessive--compulsive disorder
}

\author{
Eric Burguière, Luc Mallet
}

\section{To cite this version:}

Eric Burguière, Luc Mallet. Basic mechanisms of, and treatment planning/targets for, obsessivecompulsive disorder. New Oxford Textbook of Psychiatry, 3, Oxford University Press, pp. 976-986, 2020, 10.1093/med/9780198713005.001.0001 . hal-03098692

\section{HAL Id: hal-03098692 \\ https://hal.science/hal-03098692}

Submitted on 5 Nov 2021

HAL is a multi-disciplinary open access archive for the deposit and dissemination of scientific research documents, whether they are published or not. The documents may come from teaching and research institutions in France or abroad, or from public or private research centers.
L'archive ouverte pluridisciplinaire HAL, est destinée au dépôt et à la diffusion de documents scientifiques de niveau recherche, publiés ou non, émanant des établissements d'enseignement et de recherche français ou étrangers, des laboratoires publics ou privés. 


\title{
Basic mechanisms of, and treatment planning/targets for, obsessive-compulsive disorder
}

\author{
Eric Burguière and Luc Mallet
}

\section{Introduction}

As described in Chapter 93, obsessive-compulsive disorder (OCD) is a condition featuring obsessions (intrusive ideas) and compulsions (repetitive behaviours such as checking, washing, or iterated thought patterns) associated with high levels of anxiety, and conservative estimates suggest it may affect 2.9 million persons across the EU (that is, $0.7 \%$ prevalence) [1]. The most severe forms lead to high costs both for the individual and society as a whole [2]. While there are currently many validated pharmacological and psychotherapeutical treatments for OCD, 20-30\% of patients do not respond to them [3]. This is particularly problematic, given that $10 \%$ of these patients show severe symptoms (for example, compulsions taking $>4$ hours/day), resulting in high social and financial burdens to both families and society in general.

In this chapter, we describe some promising approaches that have been developed in recent decades to better understand the functional and pathophysiological basis of OCD at various different levels, including:

- Improved understanding of functional impairments and behavioural dimensions that most affect patients (macro-scale level).

- Characterization of dysfunctional brain activities and their related neural circuits (meso-scale level).

- Identification of specific cell type and neuronal assemblages implicated in obsessive and/or compulsive disorders (micro-scale level).

Importantly, we refer to studies carried out both on humans and animal models, providing a wider range of tools to investigate the dysfunctional neural activity associated with this condition. In particular, we consider the extracellular recording of single neurons at different brain sites during specific behaviours and also the recent development of optogenetic approaches that allow the study of neural activity with a high degree of spatial, cellular, and temporal specificity. Despite the major challenges and limitations in conducting such a translational approach across species, we believe that it offers the best means of unravelling the neural basis of psychiatric diseases $[4,5]$. Our description of studies in human is complemented and extended in Chapter 97.

We do not aim exhaustively to review all the possible therapeutic strategies to treat OCD here but will illustrate the potential of a multi-level approach using powerful, recently developed tools to develop new treatments for OCD, using some relevant examples.

\section{Expanding the toolbox to find relevant targets} for OCD

Numerous studies on OCD patients over the past two decades have consistently identified abnormal patterns of activity in one neural circuit in particular-the cortico-basal ganglia (CBG) loop [6], which is known to serve both motor and cognitive functions. Anatomical and functional studies of the CBG provide further evidence of their role in the parallel processing of sensorimotor, associative, and limbic information arising from topographically and functionally distinct cortical areas [7]; sensorimotor, associative, and limbic information appears to be segregated along a dorsolateral to ventromedial axis within the CBG (Fig. 94.1).

These circuits are used to differing extents during the learning of a behaviour (either motor or cognitive). While the ventral loopthe 'reward circuit'-is more important during initial behaviour acquisition, it is the dorsolateral loop that is crucial once learning becomes habitual $[7,8,9]$. Recent studies have supported the view that the dorsomedial and the dorsolateral striatum complement each other in the elaboration and consolidation of learnt behavioural sequences. This associative CBG loop is essential in linking contextual information with its appropriate motor response. The associative loops can therefore modulate the action of sensorimotor loops in the control of stereotyped actions $[10,11]$.

This organization within distinct functional loops has been characterized in the context of the pathophysiology of OCD through 


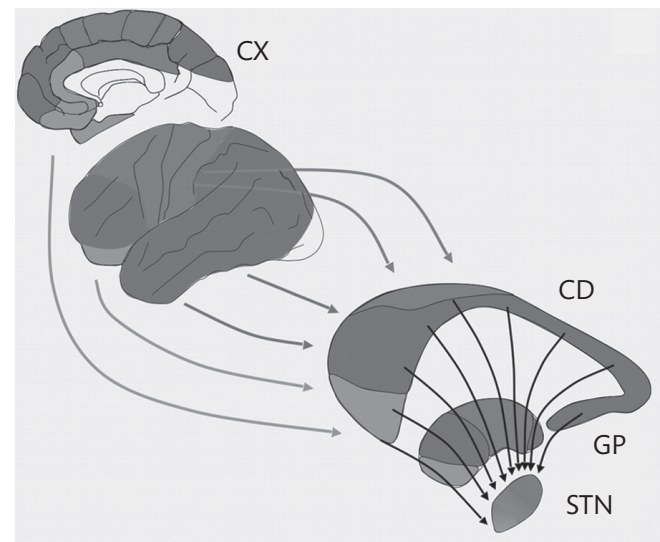

Fig. 94.1 Schematic illustration of the convergence of projections from the cerebral cortex (CX), caudate nucleus (CD), and globus pallidus (GP) in the basal ganglia. Different colours represent the types of information processed throughthe CBG: limbic (yellow), associative (violet), and sensorimotor (grefert).

Adapted from Prof Natl Acad Sci, 104(25), Mallet L, Schüpbach M, N'Diaye K, et al, Stimulation of subterritories of the subthalamic nucleus reveals its role in the The original figures had colors, pp. 10661-10666, will it be conserved in the final version? Sciences, U.S.A.

years, novel investigative techniques have emerged while more established methods have improved, allowing more detailed studies of dysfunctional brain circuits and the neural basis of OCD.

\section{Animal models}

Detailed experimental evidence on the role of the different CBG loops in OCD is scarce in human subjects, mostly due to ethical issues arising from the use of invasive procedures and the time and spatial specificity constraints inherent in non-invasive imaging techniques. For these reasons, and with the development of genetically engineered mutant mice, we have witnessed the emergence of numerous animal models relevant to OCD-like behaviours within the last decade [12]. These animal models allow detailed study of the specific mechanistic processes that could be altered in OCD treatments; the results of recent studies are promising and point to investigations of specific targets in human patients. Two recent studies have shown that in a mouse model expressing compulsive-like behaviour, compulsions could be drastically reduced by targeting the lateral orbitofrontal cortex (OFC) and the medial part of the striatum (that is, the caudate in humans) $[13,14]$. The combination of animal models of obsessive-compulsive spectrum symptoms with new methods available to neuroscientists should allow to better investigate the links between the genes implicated in OCD-related disorders and the neurophysiological circuits affected and behavioural phenotypes [13, 15-17].

The main characteristics of validating mutant animals as models of OCD are functional (OCD-related behaviour), neurophysiologic (the neural circuits affected by the mutation), and genetic (OCD candidate genes). The most promising mouse models developed so far share common behavioural phenotypes characterized by excessive grooming, which can be interpreted as a proxy for compulsive behaviour in mice, that is, an analogue of abnormal ritualistic and stereotyped behaviours that are overexpressed despite their negative consequences (for example, deleterious skin lesions). From a neural circuits point of view, there is striking evidence that a mutation in all these mouse models alters the normal functioning of the CBG circuits known to be implicated in OCD (Fig. 94.2).

The introduction of gene mutations causing symptoms related to OCD had anatomical consequences such as a decrease in striatal volume [15], but also more consistently pointed towards dysfunctional synaptic transmission, particularly in the striatum compartment (for example, equivalent to caudo-putamen areas in humans) receiving cortical inputs. These features have been shown following the deletion of genes coding for SAPAP3, Slitrk5, and EAAC1proteins located post-synaptically in striatal neurons $[13,15,18]$. These mutations had electrophysiological consequences resulting in abnormally hyperactive striatal neurons, supporting the idea that elevated striatal activity is implicated in OCD pathophysiology, as suggested by some imaging studies in human patients $[14,16]$.

\section{Imaging}

\section{Structural connectivity MRI}

Diffusion-weighted imaging (DTI) is now a well-established technique to infer brain connectivity based on magnetic resonance imaging (MRI) of the random motion of water molecules in tissues [19] (see Chapter 12). Because this motion is firmly restricted by the sheets of myelin surrounding the axons, the diffusion pattern reveals the main direction of these axons locally. The integration of information on the local directionality of white matter fibres can then be used to infer the entire network of connections within the brain using the tractography techniques now popular.

The directional dependence of molecular diffusion in white matter ('anisotropy') can be used to reconstruct tracts through the brain in vivo by assuming that the main direction of diffusion in a voxel indicates the local orientation of white matter fibres [20, 21]. A number of DTI tractography algorithms have been proposed to reconstruct fibre tracts [20]. Tractography enables the investigation of both tract-specific white matter lesions and the changes in connection probability between distinct brain regions (Fig. 94.3).

DTI has been used recently to study the pathophysiology of OCD and possible deficits in neural circuit connectivity in particular. Most of the studies have confirmed the implication of the CBG and its related fibres such as the cingulate bundle, the corpus callosum, and the anterior limb of the internal capsule [22].

Thanks to the increasing resolution and refinement of analytical algorithms in the field of anatomical imaging, it has been possible to discover new connections of interest. For example, the subthalamic nucleus (STN) has only recently been the subject of structural connectivity studies using diffusion MRI. Very few empirical data have yet been collected on the role of this nucleus in OCD, although its direct connectivity to the cortical areas, known as the hyperdirect pathway, has been implicated in action selection and decision-making [23]. A detailed connectivity map of the corticoSTN pathway in OCD patients, compared to healthy subjects, would therefore be of great interest in assessing its involvement in this condition.

With the advent of high magnetic field systems ( $>3 \mathrm{~T}$ ) capable of producing strong gradients, diffusion methods have been extended from human to small animal imaging. With such MRI systems, high resolution can be achieved (in the order of 100 microns) and very small structures in rodent brains can be visualized to study the 


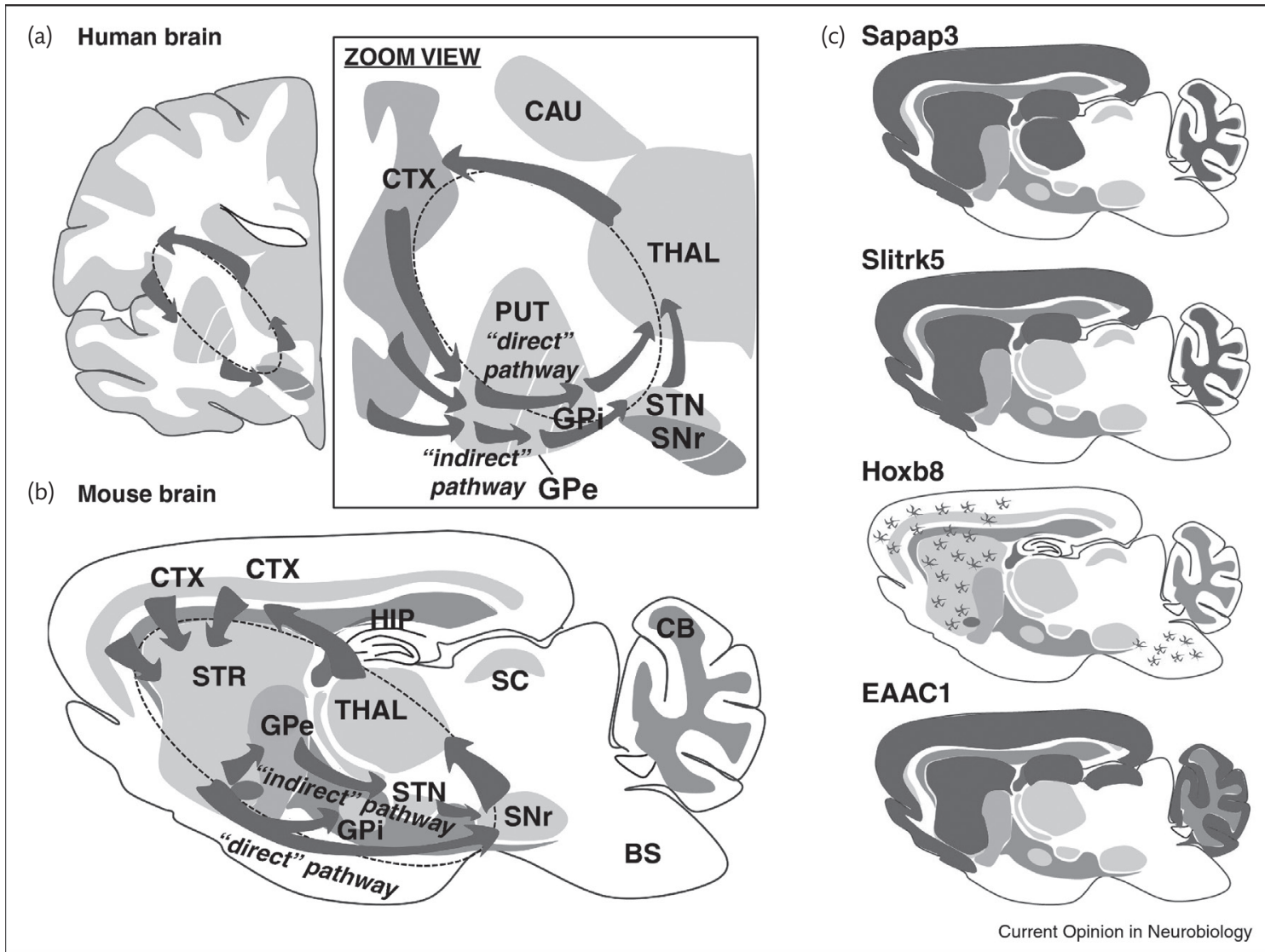

Fig. 94.2 Central role of the CSTC circuitry in obsessive-compulsive disorder in humans and compulsive-repetitive behaviours in mice. (a) Illustration of CBG loops in the human brain. (b) Illustration of equivalent CBG loops in a mouse brain. CTX, cortex; STR, striatum; CAU, caudate; PUT, putamen; HIP, hippocampus; THAL, thalamus; STN, subthalamic nucleus; SNr, substantia nigra pars reticulata; GPe, globus pallidus externa; GPi, globus pallidus interna; SC, superior colliculus; BS, brainstem; CB, cerebellum. (c) Examples of four candidate OCD gene expression patterns that have been targeted in mutant models. Note the overlapping implication of the CBG in these different expression patterns.

Reproduced from Curr Opin Neurobiol., 21(6), Ting JT, Feng G, Neurobiology of obsessive-compulsive disorder: insights into neural circuitry dysfunction through mouse genetics, pp 842-848, Copyright (2011), with permission from Elsevier Ltd.
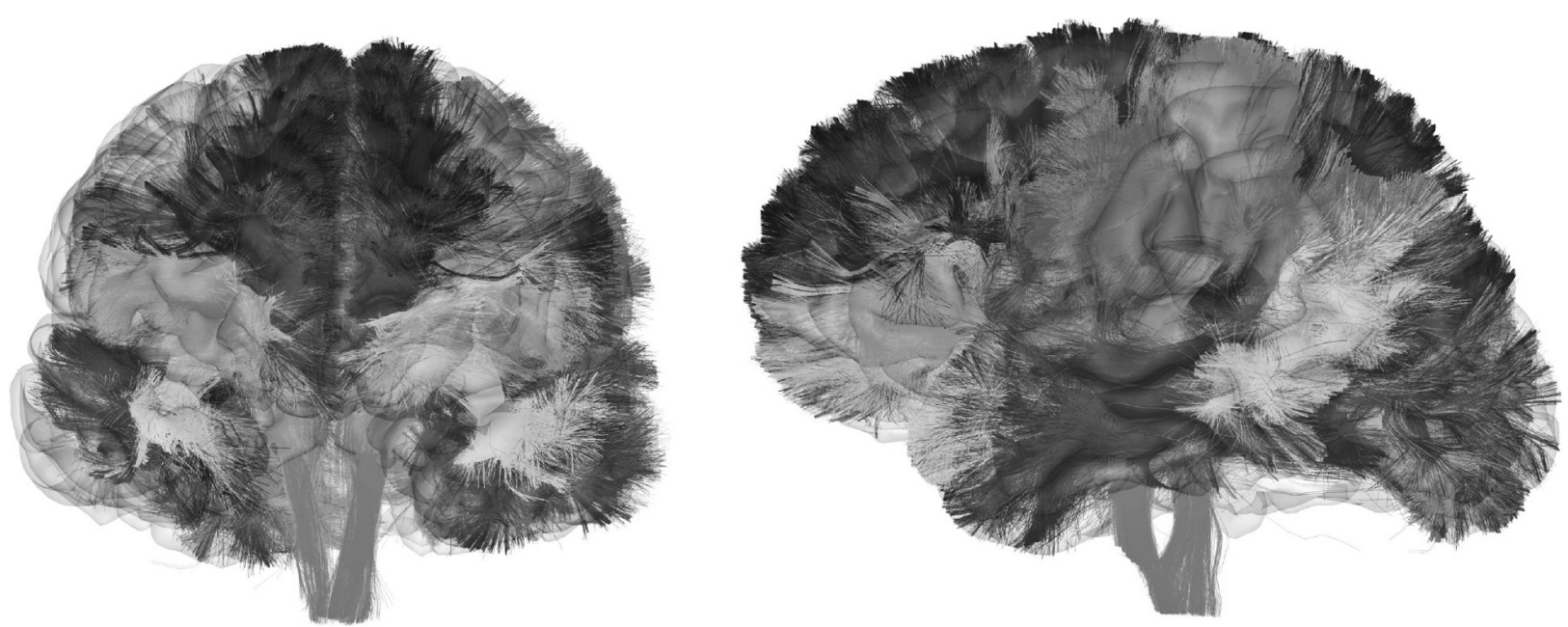

Fig. 94.3 (see Colour Plate section) CONNECT/Archi atlas of the human brain white matter connectivity developed at NeuroSpin, as part of the European CONNECT FP7 project.

Reproduced courtesy of Cyril Poupon,CEA NeuroSpin. 


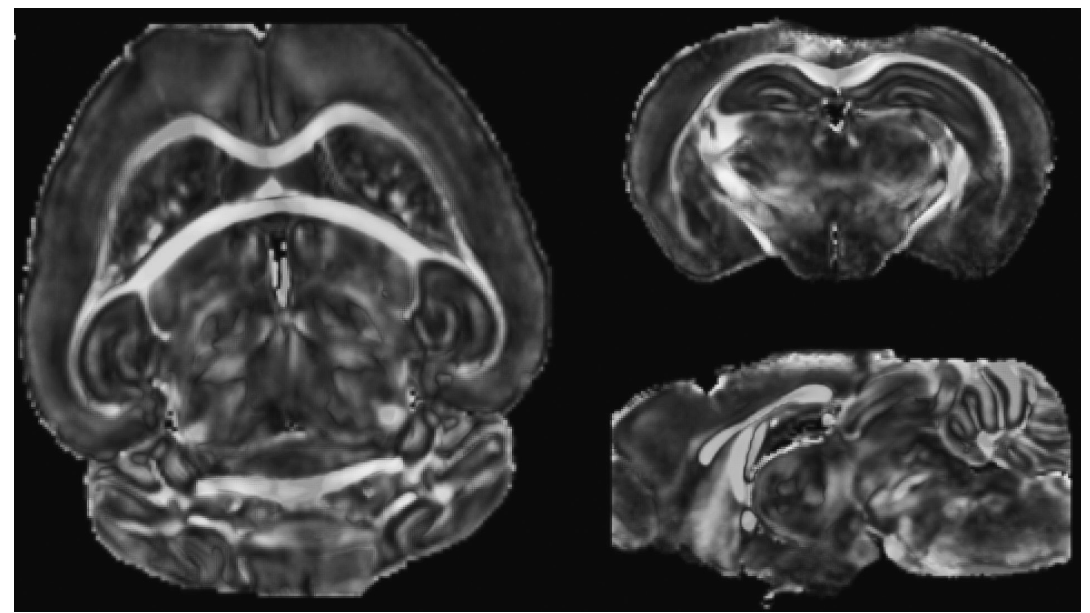

Fig. 94.4 (see Colour Plate section) Representative three-dimensional image of colour-coded fractional anisotropy in a mouse brain based on diffusion MRI at 11.7 T.

Reproduced courtesy of S. B. Seébille and M. D. Santin, ICM-CENIR.

peripheral nervous system [24], as well as neurodegenerative diseases such as multiple sclerosis, Alzheimer's disease, and Parkinson's disease (PD) [25]. Recently, feasibility studies of DTI tractography have allowed the identification of neural networks in rodent brains in target regions relating to homologous limbic and associative regions [26] (Fig. 94.4).

With the high definition offered by recent MRI systems (up to 17 $\mathrm{T}$ ), one could hope to detect potential structural differences in the CBG circuits between wildtype and animal models of OCD. One of the main advantages using anatomical connectivity in these models is that these data can be compared to the functional connectivities which can be finely characterized in animal models by using innovative investigative tools (for example, dense chronic single neurons recording and/or optogenetic neuromodulation).

\section{Functional connectivity MRI}

Once the anatomical features of a given circuit are established, the next challenge is to describe its functional aspects. Such characterization may be achieved by analysing the long-distance synchronization between structures connected by the circuit.

By using fMRI [for example, echo planar imaging (EPI)] to measure the slow changes in blood oxygenation level-dependent (BOLD) signal across distant brain regions, one can identify connected networks. This technique allows the detection of spontaneous patterns of functional connectivity MRI (fcMRI). In recent years, the fcMRI approach has identified large-scale brain networks interacting, while the subject is at rest, as well as while engaged in an active task. Across neurological and psychiatric disorders, abnormal patterns of fcMRI have been interpreted as correlates of altered brain-scale network functioning, so providing a potential marker for abnormalities in information processing. For example, recent studies of fcMRI in OCD have reported changes in functional coupling between the striatum and frontal regions, compared to healthy controls. Nakame et al. [27] found increased functional connectivity between the ventral striatum and the OFC and ventral medial prefrontal and dorsal lateral prefrontal cortices of subjects with OCD using this method. Similarly, Harrison and colleagues
$[28,29]$ consistently found that this augmented functional connectivity between the OFC and the ventral striatum was positively correlated with OCD severity. However, in the particular case of the associative-limbic hyperdirect pathway, the small volume of the STN (relatively to the typical voxel size used with functional imaging, for example $2 \mathrm{~mm}$ isotropic) poses special difficulties.

At the whole-brain scale, functional imaging of OCD points towards hyperactivity in the limbic and associative cortical regions [anterior cingulate cortex (ACC) and OFC] and related basal ganglia areas [30]. Additionally, studies from our groups and others identified abnormalities in the CBG circuits in OCD. Indeed, in fMRI studies of OCD patients, abnormal haemodynamic responses in the OFC and ACC may decrease as patients improve either through deep brain stimulation (DBS) therapies [31] or psychotherapy [32]. These data support the involvement of limbic/associative frontalbasal ganglia loops in OCD and suggest that their dysfunction might result in pathological doubt, obsessive anxiety, and compulsive behaviours.

\section{Neural recording and neuromodulation}

\section{Electrophysiology and long-range synchronization}

Unlike metabolic measures, electrophysiological recordings provide a unique source of information to assess long-distance synchronization at sub-millimetre scales with sub-millisecond resolution. Two main types of signal can be recorded-either using micro-electrodes (diameter $<50 \mu \mathrm{m}$ ), which allows the identification of spike activity, and therefore single- or multi-neuron activities; or macro-electrodes (diameter $>500 \mu \mathrm{m}$ ), which collect local field potentials (LFPs) that sum the relatively low-frequency $(<150 \mathrm{~Hz})$ electrical activity of millions of neurons in the area surrounding the tip of the electrode. The are typically used in animal studies, but during DBS neurosurgery to confirm the targeting of the intended structure. In DBS-treated patients, LFPs may be directly recorded from the definitive stimulating electrodes, while their leads are still externalized prior to connection to the pulse generator. 
To date, electrophysiological assessment of long-distance network connections has been little used in OCD patients. Most recently, simultaneous recording of the STN, LFPs, and cortical activity from scalpPlease add recent reference from our group: (ME)Mallet L, Du Montcel ST, Clair AH, Arbus C, Bardinet E, Baup N, Chabardès S, Chéreau I, e ence Czernecki V, Fontaine D, Harika-Germaneau G, Haynes WI, Houeto JL, Jaafari N, Krack P, gesti Millet B, Navarro S, Polosan M, Pelissolo A, Welter ML; STOC Inter Long-term Study Group. Long-term effects of subthalamic stimulation in Obsessivelogic Compulsive Disorder: Follow-up of a randomized controlled trial. was Brain Stimul. 2019 Jul - Aug;12(4):1080-1082. doi: 10.1016/j.brs.2019.04.004.

tients and unaffected subjects would show how neural activity within elements of the CBG pathway is altered in DBS-implanted OCD patients and provide valuable data on the functional interactions between the various brain areas. By providing insight into how limbic cortical activity is co-ordinated with subcortical activity, such analyses would be crucial in understanding the pathophysiology of OCD and begin to dissect the mechanisms by which DBS functions in OCD patients. Finally, these data could lead to novel DBS technologies to target specific pathways to normalize the information transfer along the cortico-subcortical pathway that is disrupted in OCD.

\section{Deep brain stimulation}

DBS (see Chapter 19) represents a promising type of intervention for severe, resistant forms of OCD, based principally on the serendipitous observation that DBS of the basal ganglia can reduce OCD symptoms in those patients suffering from comorbid OCD $[35,36]$. Independent studies have proposed multiple targets in the CBG for DBS in OCD, such as the anterior limb of the internal capsule and the STN [37-39] (Fig. 94.5), as an alternative to stereotactic lesional neurosurgery. But progress has been limited by the lack of understanding of the pathophysiology of OCD at both the neural and

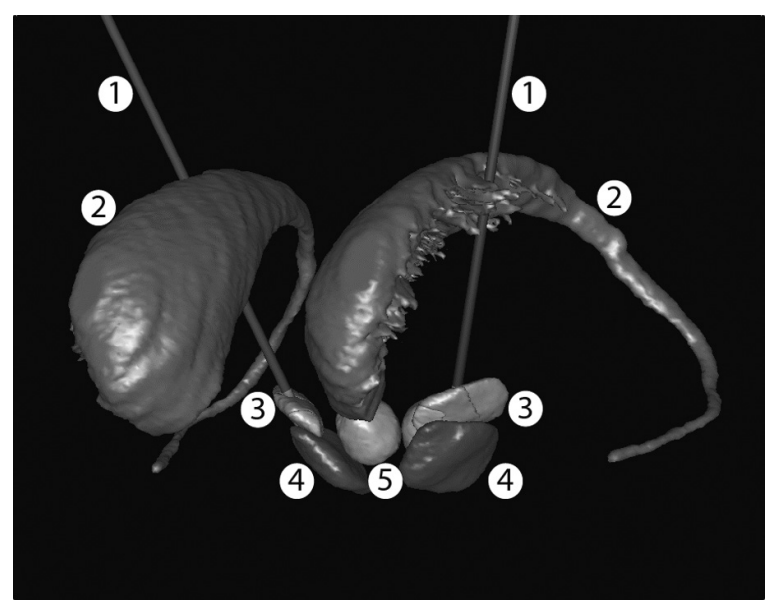

Fig. 94.5 (see Colour Plate section) Three-dimensional image of electrodes implantated in a DBS procedure. Depending on the stimulating electrode (5) configuration and stereotactic co-ordinates, several anatomical sites can be targeted. Two brain targets are represented in this example: the caudate caudate nucleus (2) and the subthalamic nucleus (2). Other brain structures, such as the substantia nigra (3) and the red nucleus (4), can be used as anatomical landmarks Adapted courtesy of Jerome Yelnik, INSERM/ICM. behavioural levels and how DBS operates on these brain circuits and their functions. Two meta-analyses have suggested that OCD symptoms showed a mean improvement of 45\% after 3-36 months

\section{$\%$
e-
ly
ly
a-
ht}

ht

m

s. d

nucleus of the stria terminalis have been associated with a median reduction of $46 \%, 36-64 \%$, and $45 \%$ in OCD severity, respectively, 36-171 months after surgery [42-45].

Understanding the contribution of cortico-subcortical circuits to OCD pathophysiology and therapeutic interventions therefore represents a major challenge in improving the selection of candidate patients and the optimization of treatment strategies. As recognized by various authors [46], traditional nosography-even when combined with more recent techniques such as brain imaging, has made limited progress in this direction, and novel approaches combining hypothesis-driven experimental studies of endophenotypes, animal models, and neuromodulation may be essential to open the way to translational research for innovative treatments.

As an example, therapeutic mechanisms underlying the action of STN high-frequency stimulation (HFS) involve the hyperdirect pathway. This pathway provides direct cortical inputs to the STN. It was first described in the motor/premotor domain and was considered to act through motor selection [23]. Recent evidence shows a limbic element stemming from the OFC and ACC [47]. Current proposals regarding DBS treatment in humans now incorporate the hyperdirect pathway not only for motor disorders, but also for psychiatric conditions such as OCD [48, 49]. Indeed, the effective target for OCD was the associative-limbic part of the STN-HFS [38], which is the target of hyperdirect afferents from the limbic and associative prefrontal regions (ACC and OFC).

Interestingly, per-operative recordings have revealed that populations of neurons from the associative-limbic part of the STN exhibit electrophysiological correlates of OCD symptoms (for example, repetitive checking [50]). At the cellular level, burst and oscillatory patterns of activity from the associative-limbic part of the STN correlate with OCD symptomatology (across patients) and predict the post-operative long-term response of STN-HFS [51].

However, the nature of the benefit is not clear and difficult to investigate in patients, as the amount of physiological data simultaneously available at each node of the cortico-subcortical loops is limited. Moreover, the use of DBS is today empirically driven. The exact effects of this technique are not known, especially the physiological consequences of such stimulations on the CBG loop [52]. Finally, electrical currents may trigger antidromic effects in afferent neurons, most notably in limbic cortical areas, paralleling the effects shown in cases of Parkinsonism [53].

\section{Optogenetics}

The functional deconstruction of neuronal circuits can now be achieved in animal models using optogenetic techniques [54]. These 
have already been used in rodents to decipher neural codes of basic motor, cognitive, and emotional behaviours $[14,53,55,56]$. These techniques allow the manipulation of neuronal activity using optical stimulation by expressing, through viral vectors, specific lightsensitive membrane proteins-called opsins-in neural cells. This approach is of great interest in the selective inhibition or excitation of nodes of given targeted circuits and can be used to assess physiological and functional causal links. Using a combination of genetic and stimulation-dependent effects, optogenetics allows a high level of temporal, spatial, and cell type specificity.

It can also be used to overcome certain issues encountered in previous classical approaches. For example, the observation of electrophysiological effects of DBS in OCD patients is challenging because of the electrical artefacts it induces. DBS effects also depend on cell type, active fibres, and nearby structures. These issues could be overcome by taking advantage of the optogenetic technology, which avoids electrical artefacts and allows the stimulation of specific cell types only.

Primary applications of optogenetics have been used to better analyse and understand discrete and specific normal or abnormal behaviours in rodents. To effectively validate and translate this novel knowledge to clinical applications, the next step is to scale up these optical experiments to animal models displaying greater anatomical and physiological similarities with human beings, especially in complex behavioural abnormalities. In particular, rodents allow for a wide range of tools to be used in order to manipulate the cortexbasal ganglia circuits, yielding models of neurologic and psychiatric disorders.

Using optogenetics in rodents, some recent studies have suggested new targets that could be considered to be causally linked to OCD symptomatology. Optogenetic manipulation of specific brain circuits, such as the orbitofrontal-striatal pathway, could induce or decrease compulsive-like behaviours [14, 57]. Although these brain areas have previously been regularly identified as abnormally active in OCD patients, these studies were the first to show their manipulation could elicit/decrease compulsive-like behaviours. Thus, an optogenetic approach in animal models not only allows the testing of circuit-level hypotheses for OCD, but also challenges further the micro-circuitry within various brain areas. Indeed, genetic studies in human patients have already indicated that genes affecting the serotonergic, dopaminergic, and glutamatergic systems, and the interaction between them, may be affected in OCD and many potential therapeutic targets may arise from these biological systems [58]. Furthermore, Burguière et al. [14] focused on a new potential cellular subtype-the striatal interneurons-which may play an essential role in regulating the expression of striatal-dependent behaviours and could be implicated in OCD (Fig. 94.6).

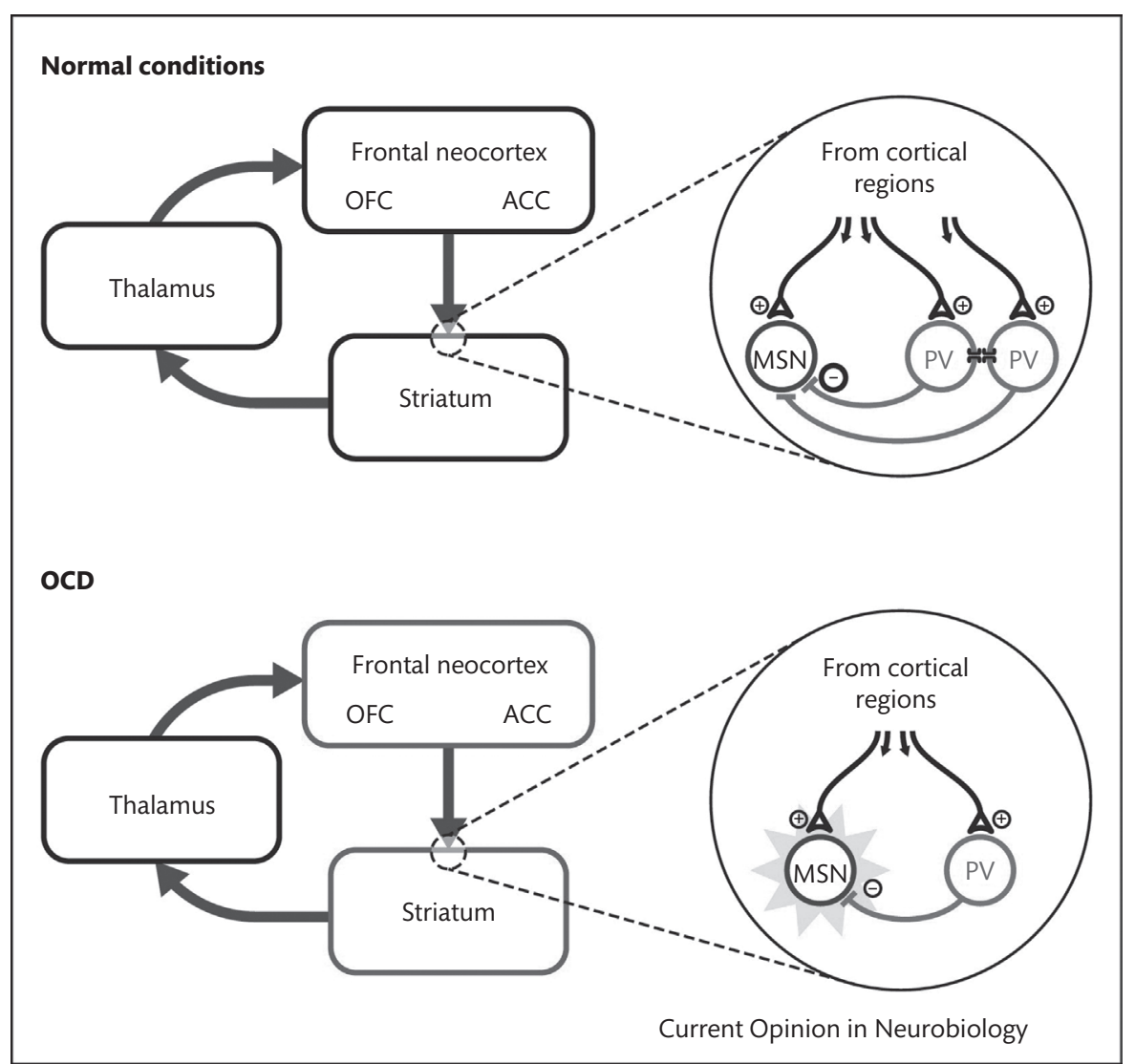

Fig. 94.6 Hypothetical model to illustrate striatal hyperactivity as a consequence of interneuronal network deficiency. Above: in normal conditions, an extensive inhibitory network in the striatum preserves a balance of excitation and inhibition driven by the cortex. Below: in the OCD condition, there is an imbalance towards excitation in the striatal region due to a lack of interneurons. As a result, there will be hyperactivation of the entire CBG loops. ACC, anterior cingulate cortex; MSN, medium spiny neurons; OFC, orbitofrontal cortex; PV, parvalbumin-positive interneurons.

Reproduced from Curr Opin Neurobiol., 30, Burguière E, Monteiro P, Mallet L, et al, Striatal circuits, habits, and implications for obsessive-compulsive disorder, pp 59-65, Copyright (2015), with permission from Elsevier Ltd. 
In fact, it has been shown that a powerful inhibitory network of parvalbumin (PV)-immunoreactive and cholinergic (Chat)immunoreactive interneurons is crucial for the functional regulation of striatal networks [59]. Moreover, depending on whether either the sensorimotor or associative-limbic cortico-striatal pathways are affected by a lack of interneurons, the functional consequences can be drastically different. According to human imaging studies, it has been suggested that cortico-striatal sensorimotor pathways (including the dorsolateral part of the striatum equivalent to the putamen in humans) are more likely to be affected in Tourette's patients, while cortico-striatal associative-limbic pathways (including the medio-ventral part of the striatum or caudate nucleus in humans) are more likely to be affected in OCD patients, although some overlap can be observed in the pathophysiology of these behaviours [60-63].

\section{Linking behavioural and neurophysiological endophenotypes of OCD to strengthen target validity}

Experimental psychologists and clinicians have performed numerous studies on OCD patients to assess their performance in the main cognitive domains (memory, attention, verbal fluency, etc.) using classical behavioural test batteries. Even if some deficits have been observed, no clear magnitudes of effects for each cognitive domain have been observed using meta-analyses [64-66]. So in order to identify the relevant behavioural dimensions that might be affected by OCD, investigators must focus on more selective cognitive processes. To illustrate this approach, we will discuss three dimensions that could prove dysfunctional in OCD, as well as the underlying brain circuits that play a role in these processes and that often overlap with those identified in the pathology.

\section{Uncertainty monitoring}

Compulsive checking is a core symptom of OCD. It is characterized by the urge to verify repeatedly that an action, usually aimed at preventing a possible harm, has been properly completed [67]. The clinical phenomenology of compulsive checking illustrates two hypotheses regarding its psychopathological mechanismscompulsive checking may proceed from a maladaptive goal-directed behaviour towards uncertainty reduction; or, in the context of habitdriven behaviours, it may result from a failure to control impulses to check. These two alternatives fit nicely into two lines of research on the behavioural functions of the basal ganglia in general, and the STN in particular.

A growing number of studies have expanded the role of the STN from purely motor functions to include higher-level processes such as emotion and decision-making [68, 69]. In PD patients (for whom STN-HFS treatment is most widespread), the STN has been associated with the regulation of behaviour in the face of conflicting stimuli [70], for example postponement when faced with two positively reinforced cues in reward-based motor tasks [70-72]. Conversely, lesion and stimulation studies in animals have also shown that various forms of STN intervention result in premature responses and increased attentional errors [73, 74]. Interestingly, the hyperdirect pathway, which connects the cortical frontal areas to the STN [23], as previously described, could constitute an anatomical route for such decisional signals whereby behaviour is regulated according to the strength of the sensory evidence. Indeed, the hyperdirect pathway not only connects motor/premotor functions, but also the limbic/ associative regions, such as the ACC to the anterior-medial STN, precisely the target of DBS in the context of OCD [47]. Considering the role of the ACC and other associative/limbic frontal cortices in action selection and decision-making, one could surmise that the STN receives higher-level decisional signals [69] to regulate a behaviour adaptively.

Recently, new behavioural paradigms have been developed to assess uncertainty monitoring in animals, revealing that, when given the opportunity, rats can adaptively 'opt out' and move on to the next, possibly easier, trial of the task $[75,76]$. In such tasks, varying uncertainty about stimulus identity (hence decision difficulty) leads the animal to discard the trials where its response is most uncertain. Indeed, simultaneous electrophysiological recordings have shown that stimulus-related uncertainty is encoded in the spike rate of the OFC neurons. However, despite putative connectivity (via the hyperdirect pathways), it is still unknown whether these uncertainty monitoring signals reach the STN and participate in the regulation of goal-directed behaviour, either to opt out from a trial or to check back at the stimulus.

Paradoxically, empirical studies in humans have reported that repetitive checking, even in healthy subjects, instead of reducing stimulus-related uncertainty, actually decreases subjective confidence [77]. To investigate repetitive checking in the laboratory, we introduced a so-called 'verification task', based on a working memory paradigm in which, on each trial, participants could review the stimulus before proceeding to the feedback. This paradigm allowed us to provoke and assess compulsive checking in OCD patients $[78,79]$ and also to reproduce this pathological behaviour during the DBS surgery when the patient is awake and behaving [50]. We were therefore able to record the STN from populations of neurons, as OCD patients entered bouts of repetitive checking. The STN showed a significant increase in the population spike rate when the patient was about to perform another checking action, compared to when he/she was about to proceed to the next trial. These results suggest that uncertainty signals anticipating checking actions may be detected in STN neurons [50].

\section{Behavioural inhibition}

A different and contrasting line of research has focused on action control $[80,81]$, comprising behavioural inhibition and the cessation of ongoing motor programmes before they have reached overt expression. According to the classical model of the basal ganglia-thalamo-cortical loops, the major output nuclei of the basal ganglia (the STN and the internal globus pallidus) maintain sustained inhibition at the cortical level via the thalamic relay. To explore behavioural inhibition in humans, clinical [35], functional [68], and electrophysiological studies in DBS-treated PD patients have proved critical, linking successful behavioural inhibition to frequency-specific changes of oscillatory activity within the STN [71], as well as between the STN and prefrontal cortices [82]. As a consequence, a lesion or inactivation of the STN performed in rats leads to impulsive behaviour, characterized by failed inhibition of prepotent responses initiated in the striatum-pallidum circuits [83]. In this context, compulsive behaviour may reflect a functional deficit at the STN level, causing failure to inhibit prepotent 
or habitual actions that are produced, irrespective of the behavioural context.

Indeed, neural representations of habitual sequences of actions triggered as encapsulated motor programmes, or 'chunks', have been observed in the striatal circuits of rodents [84-86]. Additionally, perturbation of the fronto-striatal circuitry could indeed modulate the execution of habitual actions and prevent compulsive behaviour, as has been shown using optogenetic manipulation in mice [14] and DBS in OCD patients [31]. Therefore, the fronto-striato-pallidal pathway may play an important role in the emergence of motor programmes and, if dysfunctional, provoke their overexpression, leading to compulsive behaviours. Complementary to the corticostriatal pathway, the cortico-STN pathway (that is, the hyperdirect pathway) has been shown to stop habitual behaviours before they reach completion [82, 87]. In this context, compulsive behaviour may reflect a functional deficit which fails to inhibit prepotent or habitual actions that are produced, irrespective of the behavioural context.

\section{Behavioural flexibility}

OCD in humans is characterized by repetitive behaviour, performed through rigid rituals. This phenomenological observation has led to exploration of the idea that OCD patients have diminished behavioural flexibility (that is, the ability to change one's behaviour according to contextual cues). Behavioural flexibility may be challenged in experimental tasks such as reversal learning paradigms. In these tasks, the participant has to respond to either of two visual stimuli. In the deterministic version, one stimulus is positively rewarded while the other is not. Unbeknown to the participant, the reward contingencies are reversed after a certain number of trials, so that the previously neutral stimulus is now rewarded while the previously rewarded stimulus is not. In this deterministic version, healthy adult humans immediately detect the change in contingencies and start responding to the other stimulus. In addition, the task may be rendered more challenging using a probabilistic version of it [88]. Here, the reward is not systematically delivered after the subject has chosen the rewarding stimulus, providing uncertainty regarding the current state of the reward contingencies. Only after having collected enough evidence that the previously reinforced stimulus has changed, will the participant shift to the newly (but still varyingly) reinforced stimulus. Performance in reversal learning is scored according to the number of persistent errors committed when participants maintain their response towards previously reinforced stimuli in spite of a negative reward. At the brain level, behavioural flexibility tasks, and reversal learning in particular, appear to engage the OFC and ACC regions. In a 2006 study, Remijnse et al. [89] showed that OCD was associated with a reduced response in the orbitofrontal-striatal network during adaptive switching to the new stimulus reinforcement regime. Using a different type of behavioural flexibility task, Chamberlain et al. [90] has demonstrated that OCD patients, as well as their relatives, show diminished OFC responses to behavioural adaptation triggered by the task, pointing towards the existence of an OCD endophenotype. Interestingly, in PD, STN stimulation has a beneficial effect on mental flexibility and working memory [91] and on cognitive flexibility, as expressed by the inhibition of habitual responses tested in a random number generation task [92]. Unsurprisingly, similar behavioural tasks have long been used with mice using various response modalities (T-maze, lever press, nose-poke), and animal studies have confirmed the role of $\mathrm{OFC}$ or OFC homologous regions in behavioural flexibility.

\section{Conclusions}

Within the last decade, a multi-dimensional approach has been proposed to better link clinical observations with the increasing amount of data obtained in biological psychiatry studies [93, 94].

In fact, the integrative view of complementary research fields, including neuroimaging, genetics, experimental psychology, computational psychiatry, and animal models, has led to a redefinition of OCD nosography and its heterogenous expression. In the future, further insights may come from studies combining different fields, as illustrated by recent pioneering work with OCD patients which has combined computational and neurophysiological approaches [95] or experimental psychology with neuroimaging [96]. The identification of new targets using such complementary approaches could also be used to develop entirely novel animal models of psychiatric diseases. It would open the way to developing new ideas by manipulating brain circuits, for example using optogenetic manipulations that would reproduce relevant aspects of the OCD symptomatology.

To analyse and understand abnormal behavioural functions in OCD patients, a promising perspective comes from the recent field of computational psychiatry. It sets out to investigate the changes in elementary cognitive processes in dysfunctional patients, such as behavioural flexibility or uncertainty monitoring, as described previously. Using the computational approach, researchers try to infer the hidden variables and states associated with the computations the brain might use when performing a task. Indeed, mathematical models of how choices emerge from elementary computations can potentially bridge the gap between neural activities and the behavioural choices.

A more translational and comprehensive approach that integrates novel fields of fundamental and clinical research should therefore be used to unveil the neurobiology of OCD and possibly lead to the development of more effective treatments.

\section{REFERENCES}

1. Wittchen HU, Jacobi F, Rehm J, et al. The size and burden of mental disorders and other disorders of the brain in Europe 2010. Eur Neuropsychopharmacol 2011, 21:655-79.

2. World Health Organization. The World Health Report 2004: Changing History. Geneva: World Health Organization; 2004.

3. Abramowitz JS, Taylor S, McKay D. Obsessive-compulsive disorder. Lancet 2009, 374:491-9.

4. Insel TR. From animal models to model animals. Biol Psychiatry 2007, 62:1337-9.

5. Nestler EJ, Hyman SE. Animal models of neuropsychiatric disorders. Nat Neurosci 2010, 13:1161-9.

6. Saxena S, Rauch SL. Functional neuroimaging and the neuroanatomy of obsessive-compulsive disorder. Psychiatr Clin North Am 2000, 23:563-86.

7. Alexander GE, DeLong MR, Strick PL. Parallel organization of functionally segregated circuits linking basal ganglia and cortex. Annu Rev Neurosci 1986, 9:357-81. 
8. Graybiel AM. Habits, rituals, and the evaluative brain. Annu Rev Neurosci 2008, 31:359-87.

9. Yin $\mathrm{HH}$, Knowlton BJ. The role of the basal ganglia in habit formation. Nat Rev Neurosci 2006, 7:464-76.

10. Thorn CA, Atallah H, Howe M, Graybiel AM. Differential dynamics of activity changes in dorsolateral and dorsomedial striatal loops during learning. Neuron 2010, 66:781-95.

11. Yin HH, Mulcare SP, Hilário MRF, et al. Dynamic reorganization of striatal circuits during the acquisition and consolidation of a skill. Nat Neurosci 2009, 12:333-41.

12. Ting JT, Feng G. Neurobiology of obsessive-compulsive disorder: insights into neural circuitry dysfunction through mouse genetics. Curr Opin Neurobiol 2011, 21:842-8.

13. Welch JM, Lu J, Rodriguiz RM, et al. Cortico-striatal synaptic defects and OCD-like behaviours in Sapap3-mutant mice. Nature 2007, 448:894-900.

14. Burguière E, Monteiro P, Feng G, Graybiel AM. Optogenetic stimulation of lateral orbitofronto-striatal pathway suppresses compulsive behaviours. Science 2013, 340:1243-6.

15. Shmelkov SV, Hormigo A, Jing D, et al. Slitrk5 deficiency impairs corticostriatal circuitry and leads to obsessive-compulsive-like behaviours in mice. Nat Med 2010, 16:598-602.

16. Burguière E, Monteiro P, Mallet L, Feng G, Graybiel AM. Striatal circuits, habits, and implications for obsessive-compulsive disorder. Curr Opin Neurobiol 2015, 30:59-65.

17. Greer JM, Capecchi MR. Hoxb8 is required for normal grooming behaviour in mice. Neuron 2002, 33:23-34.

18. Aoyama K, Suh SW, Hamby AM, et al. Neuronal glutathione deficiency and age-dependent neurodegeneration in the EAAC1 deficient mouse. Nat Neurosci 2006, 9:119-26.

19. Le Bihan D. Looking into the functional architecture of the brain with diffusion MRI. Nat Rev Neurosci 2003, 4:469-80.

20. Mori S, Crain BJ, Chacko VP, van Zijl PC. Three-dimensional tracking of axonal projections in the brain by magnetic resonance imaging. Ann Neurol 1999, 45:265-9.

21. Poupon C, Clark CA, Frouin V, et al. Regularization of diffusionbased direction maps for the tracking of brain white matter fascicles. NeuroImage 2000, 12:184-95.

22. Koch K, Reess TJ, Rus OG, Zimmer C, Zaudig M. Diffusion tensor imaging (DTI) studies in patients with obsessive-compulsive disorder (OCD): a review. J Psychiatr Res 2014, 54:26-35.

23. Nambu A, Tokuno H, Takada M. Functional significance of the cortico-subthalamo-pallidal 'hyperdirect' pathway. Neurosci Res 2002, 43:111-17.

24. Takagi T, Nakamura M, Yamada M, et al. Visualization of peripheral nerve degeneration and regeneration: monitoring with diffusion tensor tractography. NeuroImage 2009, 44:884-92.

25. Soria G, Aguilar E, Tudela R, Mullol J, Planas AM, Marin C. In vivo magnetic resonance imaging characterization of bilateral structural changes in experimental Parkinson's disease: a T2 relaxometry study combined with longitudinal diffusion tensor imaging and manganese-enhanced magnetic resonance imaging in the 6hydroxydopamine rat model. Eur J Neurosci 2011, 33:1551-60.

26. Gutman DA, Keifer OP, Magnuson ME, et al. A DTI tractography analysis of infralimbic and prelimbic connectivity in the mouse using high-throughput MRI. NeuroImage 2012, 63:800-11.

27. Nakamae T, Sakai Y, Abe Y, et al. Altered fronto-striatal fiber topography and connectivity in obsessive-compulsive disorder. PLoS One. 2014, 9:e112075.

28. Harrison BJ, Soriano-Mas C, Pujol J, et al. Altered corticostriatal functional connectivity in obsessive-compulsive disorder. Arch Gen Psychiatry 2009, 66:1189-200.
29. Harrison BJ, Pujol J, Cardoner N, et al. Brain corticostriatal systems and the major clinical symptom dimensions of obsessivecompulsive disorder. Biol Psychiatry 2013, 73:321-8.

30. Menzies L, Achard S, Chamberlain SR, et al. Neurocognitive endophenotypes of obsessive-compulsive disorder. Brain 2007, 130:3223-36

31. Figee M, Luigjes J, Smolders R, et al. Deep brain stimulation restores frontostriatal network activity in obsessive-compulsive disorder. Nat Neurosci 2013, 16:386-7.

32. Morgiève M, N'Diaye K, Haynes WIA, et al. Dynamics of psychotherapy-related cerebral haemodynamic changes in obsessive compulsive disorder using a personalized exposure task in functional magnetic resonance imaging. Psychol Med 2014, 44:1461-73.

33. Hirschmann J, Özkurt TE, Butz M, et al. Distinct oscillatory STNcortical loops revealed by simultaneous MEG and local field potential recordings in patients with Parkinson's disease. NeuroImage 2011, 55:1159-68

34. Williams D, Tijssen M, Van Bruggen G, et al. Dopamine-dependent changes in the functional connectivity between basal ganglia and cerebral cortex in humans. Brain J Neurol 2002, 125:1558-69.

35. Mallet L, Mesnage V, Houeto J-L, et al. Compulsions, Parkinson's disease, and stimulation. Lancet 2002, 360:1302-4.

36. Nuttin B, Cosyns P, Demeulemeester H, Gybels J, Meyerson B. Electrical stimulation in anterior limbs of internal capsules in patients with obsessive-compulsive disorder. Lancet 1999, 354:1526.

37. Nuttin BJ, Gabriëls LA, Cosyns PR, et al. Long-term electrical capsular stimulation in patients with obsessive-compulsive disorder. Neurosurgery 2008, 62:966-77.

38. Mallet L, Polosan M, Jaafari N, et al. Subthalamic nucleus stimulation in severe obsessive-compulsive disorder. N Engl J Med 2008, 359:2121-34.

39. Greenberg BD, Gabriels LA, Malone DA Jr, et al. Deep brain stimulation of the ventral internal capsule/ventral striatum for obsessive-compulsive disorder: worldwide experience. $\mathrm{Mol}$ Psychiatry 2010, 15:64-79.

40. Kisely S, Hall K, Siskind D, Frater J, Olson S, Crompton D. Deep brain stimulation for obsessive-compulsive disorder: a systematic review and meta-analysis. Psychol Med 2014, 44:3533-42.

41. Alonso P, Cuadras D, Gabriëls L, et al. Deep brain stimulation for obsessive-compulsive disorder: a meta-analysis of treatment outcome and predictors of response. PLoS One 2015, 10:e0133591.

42. Ooms P, Mantione M, Figee M, Schuurman PR, van den Munckhof P, Denys D. Deep brain stimulation for obsessive-compulsive disorders: long-term analysis of quality of life. J Neurol Neurosurg Psychiatry 2014, 85:153-8.

43. Greenberg BD, Malone DA, Friehs GM, et al. Three-year outcomes in deep brain stimulation for highly resistant obsessivecompulsive disorder. Neuropsychopharmacol 2006, 31:2384-93.

44. Luyten L, Hendrickx S, Raymaekers S, Gabriëls L, Nuttin B. Electrical stimulation in the bed nucleus of the stria terminalis alleviates severe obsessive-compulsive disorder. Mol Psychiatry 2016, 21:1272-80

45. Fayad SM, Guzick AG, Reid AM, et al. Six-nine year follow-up of deep brain stimulation for obsessive-compulsive disorder. PLoS One 2016, 11:e0167875.

46. Rasmussen SA, Eisen JL, Greenberg BD: Toward a neuroanatomy of obsessive-compulsive disorder revisited. Biol Psychiatry 2013, 73:298-9.

47. Haynes WIA, Haber SN. The organization of prefrontalsubthalamic inputs in primates provides an anatomical substrate for both functional specificity and integration: implications for 
basal ganglia models and deep brain stimulation. J Neurosci 2013, 33:4804-14.

48. Le Jeune F, Vérin M, N’Diaye K, et al. Decrease of prefrontal metabolism after subthalamic stimulation in obsessive-compulsive disorder: a positron emission tomography study. Biol Psychiatry 2010, 68:1016-22.

49. Haynes WIA, Mallet L. High-frequency stimulation of deep brain structures in obsessive-compulsive disorder: the search for a valid circuit. Eur J Neurosci 2010, 32:1118-27.

50. Burbaud P, Clair A-H, Langbour N, et al. Neuronal activity correlated with checking behaviour in the subthalamic nucleus of patients with obsessive-compulsive disorder. Brain J Neurol 2013, 136:304-17.

51. Welter M-L, Burbaud P, Fernandez-Vidal S, et al. Basal ganglia dysfunction in OCD: subthalamic neuronal activity correlates with symptoms severity and predicts high-frequency stimulation efficacy. Transl Psychiatry 2011, 1:e5.

52. McIntyre CC, Grill WM, Sherman DL, Thakor NV. Cellular effects of deep brain stimulation: model-based analysis of activation and inhibition. J Neurophysiol 2004, 91:1457-69.

53. Gradinaru V, Mogri M, Thompson KR, Henderson JM, Deisseroth K. Optical deconstruction of parkinsonian neural circuitry. Science 2009, 324:354-9.

54. Deisseroth K. Optogenetics. Nat Methods 2011, 8:26-9.

55. Tye KM, Prakash R, Kim S-Y, et al. Amygdala circuitry mediating reversible and bidirectional control of anxiety. Nature 2011, 471:358-62.

56. Yizhar O, Fenno LE, Prigge M, et al. Neocortical excitation/inhibition balance in information processing and social dysfunction. Nature 2011, 477:171-8.

57. Ahmari SE, Spellman T, Douglass NL, et al. Repeated corticostriatal stimulation generates persistent OCD-like behaviour. Science 2013, 340:1234-9.

58. Pauls DL, Abramovitch A, Rauch SL, Geller DA. Obsessivecompulsive disorder: an integrative genetic and neurobiological perspective. Nat Rev Neurosci 2014, 15:410-24.

59. Kreitzer AC. Physiology and pharmacology of striatal neurons. Annu Rev Neurosci 2009, 32:127-47.

60. Beucke JC, Sepulcre J, Talukdar T, et al. Abnormally high degree connectivity of the orbitofrontal cortex in obsessive-compulsive disorder. JAMA Psychiatry 2013, 70:619-29.

61. Lewis M, Kim S-J. The pathophysiology of restricted repetitive behaviour. J Neurodev Disord 2009, 1:114-32.

62. Rotge J-Y, Guehl D, Dilharreguy B, et al. Meta-analysis of brain volume changes in obsessive-compulsive disorder. Biol Psychiatry 2009, 65:75-83.

63. Worbe Y, Mallet L, Golmard J-L, et al. Repetitive behaviours in patients with Gilles de la Tourette syndrome: tics, compulsions, or both? PLoS One 2010, 5:e12959.

64. Shin NY, Lee TY, Kim E, Kwon JS. Cognitive functioning in obsessive-compulsive disorder: a meta-analysis. Psychol Med 2013, 44:1121-30.

65. Abramovitch A, Abramowitz JS, Mittelman A. The neuropsychology of adult obsessive-compulsive disorder: a meta-analysis. Clin Psychol Rev 2013, 33:1163-71.

66. Snyder HR, Kaiser RH, Warren SL, Heller W. Obsessivecompulsive disorder is associated with broad impairments in executive function: a meta-analysis. Clin Psychol Sci 2015, 3:301-30.

67. Rachman S. A cognitive theory of compulsive checking. Behav Res Ther 2002, 40:625-39.
68. Mallet L, Schüpbach M, N'Diaye K, et al. Stimulation of subterritories of the subthalamic nucleus reveals its role in the integration of the emotional and motor aspects of behaviour. Proc Natl Acad Sci U S A 2007, 104:10661-6.

69. Weintraub DB, Zaghloul KA. The role of the subthalamic nucleus in cognition. Rev Neurosci 2013, 24:125-38.

70. Frank MJ. Hold your horses: a dynamic computational role for the subthalamic nucleus in decision making. Neural Netw 2006, 19:1120-36

71. Brittain J-S, Brown P. Oscillations and the basal ganglia: motor control and beyond. NeuroImage 2014, 85 Pt 2:637-47.

72. Zaghloul KA, Weidemann CT, Lega BC, Jaggi JL, Baltuch GH, Kahana MJ. Neuronal activity in the human subthalamic nucleus encodes decision conflict during action selection. J Neurosci 2012, 32:2453-60.

73. Baunez C, Lardeux S. Frontal cortex-like functions of the subthalamic nucleus. Front Syst Neurosci 2011, 5:83.

74. Coulthard EJ, Bogacz R, Javed S, et al. Distinct roles of dopamine and subthalamic nucleus in learning and probabilistic decision making. Brain 2012, 135:3721-34.

75. Foote AL, Crystal JD. Metacognition in the rat. Curr Biol 2007, 17:551-5

76. Kepecs A, Uchida N, Zariwala HA, Mainen ZF. Neural correlates, computation and behavioural impact of decision confidence. Nature 2008, 455:227-31.

77. van den Hout M, Kindt M. Repeated checking causes memory distrust. Behav Res Ther 2003, 41:301-16.

78. Rotge JY, Clair AH, Jaafari N, et al. A challenging task for assessment of checking behaviours in obsessive-compulsive disorder. Acta Psychiatr Scand 2008, 117:465-73.

79. Clair AH, N'Diaye K, Baroukh T, et al. Excessive checking for non-anxiogenic stimuli in obsessive-compulsive disorder. Eur Psychiatry J 2013, 28:507-13.

80. Mink JW. The basal ganglia: focused selection and inhibition of competing motor programs. Prog Neurobiol 1996, 50:381-425.

81. Ridderinkhof KR, Ullsperger M, Crone EA, Nieuwenhuis S. The role of the medial frontal cortex in cognitive control. Science 2004, 306:443-7.

82. Alegre M, Lopez-Azcarate J, Obeso I, et al. The subthalamic nucleus is involved in successful inhibition in the stop-signal task: a local field potential study in Parkinson's disease. Exp Neurol 2013, 239:1-12.

83. Eagle DM, Baunez C, Hutcheson DM, Lehmann O, Shah AP, Robbins TW. Stop-signal reaction-time task performance: role of prefrontal cortex and subthalamic nucleus. Cereb Cortex 2008, 18:178-88.

84. Barnes TD, Kubota Y, Hu D, Jin DZ, Graybiel AM. Activity of striatal neurons reflects dynamic encoding and recoding of procedural memories. Nature 2005, 437:1158-61.

85. Jin X, Costa RM. Start/stop signals emerge in nigrostriatal circuits during sequence learning. Nature 2010, 466:457-62.

86. Cui G, Jun SB, Jin X, et al. Concurrent activation of striatal direct and indirect pathways during action initiation. Nature 2013, 494:238-42.

87. Anzak A, Gaynor L, Beigi M, et al. Subthalamic nucleus gamma oscillations mediate a switch from automatic to controlled processing: a study of random number generation in Parkinson's disease. NeuroImage 2013, 64:284-9.

88. Cools R, Clark L, Owen AM, Robbins TW. Defining the neural mechanisms of probabilistic reversal learning using event-related functional magnetic resonance imaging. J Neurosci 2002, 22:4563-7. 
89. Remijnse PL, Nielen MMA, van Balkom AJLM, et al. Reduced orbitofrontal-striatal activity on a reversal learning task in obsessivecompulsive disorder. Arch Gen Psychiatry 2006, 63:1225-36.

90. Chamberlain SR, Fineberg NA, Blackwell AD, et al. Motor inhibition and cognitive flexibility in obsessive-compulsive disorder and trichotillomania. Am J Psychiatry 2006, 163:1282-4.

91. Jahanshahi M, Ardouin CMA, Brown RG, et al. The impact of deep brain stimulation on executive function in Parkinson's disease. Brain 2000, 123:1142-54.

92. Witt K, Pulkowski U, Herzog J, et al. Deep brain stimulation of the subthalamic nucleus improves cognitive flexibility but impairs response inhibition in parkinson disease. Arch Neurol 2004, 61:697-700.
93. Mataix-Cols D, Rosario-Campos MC, Leckman JF. A multidimensional model of obsessive-compulsive disorder. Am J Psychiatry 2005, 162:228-38.

94. Katerberg H, Delucchi KL, Stewart SE, et al. Symptom dimensions in OCD: item-level factor analysis and heritability estimates. Behav Genet 2010, 40:505-17.

95. Palminteri S, Clair A-H, Mallet L, Pessiglione M. Similar improvement of reward and punishment learning by serotonin reuptake inhibitors in obsessive-compulsive disorder. Biol Psychiatry 2012, 72:244-50.

96. Chamberlain SR, Menzies L, Hampshire A, et al. Orbitofrontal dysfunction in patients with obsessive-compulsive disorder and their unaffected relatives. Science 2008, 321:421-2. 